\title{
HOLOMORPHIC APPROXIMATION ON REAL SUBMANIFOLDS OF A COMPLEX MANIFOLD ${ }^{1}$
}

\author{
BY RICARDO NIRENBERG AND R. O. WELLS, JR.
}

Communicated by John Wermer, January 4, 1967

Let $X$ be a complex manifold, let $\mathcal{O}$ be the sheaf of germs of holomorphic functions on $X$, and let $\Gamma(K, \mathcal{\theta})$ denote the ring of sections over $K \subset X$ (holomorphic functions on $K$, see [5] as a general reference for the terminology used here). If $K$ is a compact set in $X$, let $C(K)$ be the Banach algebra of continuous complex-valued functions on $K$ with respect to the maximum norm on $K$. Let $A(K)$ denote the closure of $\Gamma(K, \mathcal{O})$ in $C(K)$, and if $U \supset K$, let $A(K, U)$ denote the closure in $C(K)$ of the restriction of $\Gamma(U, \mathcal{\theta})$ to $K$.

It is an old problem to try to determine under what conditions $A(K)=C(K)$ or $A(K, U)=C(K)$, for some $U$. For instance, if $X=C^{n}$ $=U$, then $A\left(K, \boldsymbol{C}^{n}\right)$ is the polynomial algebra important in the theory of polynomial approximation, and sets for which $A\left(K, \mathrm{C}^{n}\right)$ $=C(K)$ are necessarily polynomially convex. Also one is interested in the nature of the spectrum of $A(K)$ or $A(K, U)$ which can be interpreted as a holomorphic hull of some type, depending on what $U$ is. This leads to the study of holomorphic or polynomial convexity of sets (the property of being equal to the spectrum or maximal ideal space of the algebra) and approximation theorems always give information about the relation of the various spectra involved (see $[1],[11],[12])$. In this note we announce certain results on approximation of continuous functions, in the case where $K$ is a compact real submanifold of $X$ (differentiable), with or without boundary.

Let $M$ be a real submanifold $\left(C^{\infty}\right)$ of $X$. Let $T(X)$ be the tangent bundle to $X$, and let $J: T(X) \rightarrow T(X)$ be the canonical involution $\left(J^{2}=-I\right)$ given by the complex structure on $X$. Let $T(M)$ be the tangent bundle to $M$, which is, in a natural way, a real subbundle of $T(X) \mid M$. Set

$$
H_{p}(M)=J T_{p}(M) \cap T_{p}(M), \quad p \in M .
$$

Then $H_{p}(M)$ is a complex subspace of $T_{p}(X)$, and is called the holomorphic tangent space to $M$ at $p$. We will denote by $m_{p}(M)$ the complex dimension of $H_{p}(M)$.

${ }^{1}$ Research supported by NSF Grant No. GP-5951 and Army DA-ARO-D-31124-G866. 
Our major results are the following theorems, the proofs of which will appear elsewhere.

THEOREM 1. Let $M$ be a compact (without boundary) real $C^{\infty}$ submanifold of $X$, and suppose $m_{p}(M) \equiv 0$ on $M$, then there exists an open strictly pseudoconvex domain $U$ containing $M$ such that

$$
A(M, U)=A(M)=C(M) .
$$

REMARK. This theorem was proven for $\operatorname{dim} M=2$ in [3] and for real-analytic $M$ in [10].

TheOREM $2 .{ }^{2}$ Let $M^{\prime}$ be a submanifold of $X$, with $m_{p}\left(M^{\prime}\right) \equiv 0$ on $M^{\prime}$, and let $M$ be a relatively compact open submanifold of $M^{\prime}$ with a smooth boundary, $\partial M$. Then there exists an open strictly pseudoconvex domain $U$ in $X, U \supset \bar{M}$, such that

$$
A(\bar{M}, U)=A(\bar{M})=C(\bar{M}) .
$$

Let $C^{\infty}(K)$ denote the ring of $C^{\infty}$ functions on $K \subset X$, defined by restriction. Let $D$ be a relatively compact domain in $X$ with a smooth boundary, $\partial D$, and let $M=\bar{D}$. Let $A_{d}(M)$ be the closure of $C^{\infty}(M) \cap \Gamma(D, \mathcal{\theta})$ in $C(M)$. It is clear that we have

$$
A(M) \subset A_{d}(M) \subset C(M) \cap \Gamma(D, \Theta) \subset C(M),
$$

where $C(M) \cap \Gamma(D, \theta)$ is a closed proper subalgebra of $C(M)$, which we shall denote by $A_{c}(M)$ (holomorphic functions in $D$, continuous up to the boundary). An old question has been to determine when $A(M)=A_{c}(M)$. Here we can give a partial answer to that question by proving that under certain circumstances $A(M)=A_{d}(M)$, leaving open the question of whether $A_{d}(M)=A_{c}(M)$.

Theorem 3. Let $D$ be a relatively compact strictly pseudoconvex domain in $X$ with a smooth boundary. Then $A(\bar{D})=A_{d}(\bar{D})$.

REMARK. It is likely that in this case $A_{d}(\bar{D})=A_{c}(\bar{D})$, and the question can be reduced to proving that weak $L^{2}$ solutions of $\bar{\partial}_{b} f=0$ (see [8]) on $\partial D$ can be uniformly approximated by $C^{1}$ solutions.

In the following theorem we have local polynomial convexity for submanifolds with $m_{p} \equiv 0$.

THEOREM 4. Let $M^{\prime}$ be a submanifold of $C^{n}$ with $m_{p}\left(M^{\prime}\right) \equiv 0$ near a point $p_{0} \in M^{\prime}$. Then there exists a neighborhood $U$ of $p_{0}$ in $\mathbf{C}^{n}$ such that if we set $M=M^{\prime} \cap U$, then we have

$$
A\left(\bar{M}, C^{n}\right)=C(\bar{M}),
$$

2 Theorem 2 was brought to our attention by Hugo Rossi. 
and, as a consequence, $\bar{M}$ is polynomially convex.

At this point a remark should be made about the hypothesis $m_{p}(M) \equiv 0$ in all of these theorems. It has been conjectured in [11] that submanifolds (compact) of $X$ with $m_{p}(M)>0$, (assuming constant rank, for instance) have a nontrivial holomorphic hull, i.e., the spectrum of $A(M)$, for such a manifold $M$, is necessarily larger than $M$ itself, which would imply that there is no approximation. This conjecture has been proven for a certain class of dimensions (see [11]) and some of this work has recently been extended by Greenfield (oral communication).

The proofs of the theorems are modifications of the proof of Theorem 1 , which we outline here. ${ }^{3}$ First one has to show that the hypothesis that $m_{p} \equiv 0$ on $M$ implies the existence of a strongly plurisubharmonic function defined in a neighborhood $U$ of $M$ such that $M=[\phi=0]$. The argument is local and then one uses a partition of unity (cf. [10]). Then one shows that if $f \in C^{\infty}(M)$, there exists a $u \in C^{\infty}(U)$ such that $u=f$ on $M$ and $\alpha=\bar{\partial} u$ vanishes to high order on $M$. For sufficiently small $\epsilon>0$, the tube about $M$ given by $E_{\mathrm{e}}=[\phi<\epsilon]$ is a strictly pseudoconvex domain in $X$, and we can use the solution of the $\bar{\partial}$-Neumann problem $([6],[7],[9])$ to solve the equation $\bar{\partial} v_{6}=\alpha$, and obtain the estimates

$$
\left\|v_{\epsilon}\right\|_{s} \leqq C_{s, \epsilon}\|\alpha\|_{s+1}
$$

where \|\|$_{s}$ denotes the Sobolev norms on $E_{\mathrm{e}}$, and $C_{s, \mathrm{e}}$ are constants depending on $s$ and $\epsilon$. One can show that for $s$ fixed

$$
C_{8, \epsilon}=O\left(\epsilon^{-\nu}\right)
$$

for some positive integer $\nu$. We therefore have $h_{\epsilon}=u-v_{0}$ is holomorphic in $E_{\mathrm{e}}$ since $\bar{\partial} u=\bar{\partial} v_{\mathrm{e}}=\alpha$. Thus we have that $h_{\mathrm{e}}-f$ on $M$ is given by $-v_{\epsilon}$, so to know that $h_{\mathrm{e}}$ is a uniform approximation of $f$ on $M$ we must show that $v_{e}$ is uniformly small on $M$ for some $\epsilon$. By Sobolev's lemma, there is an $s$ and a $K_{\epsilon}$ (independent of $\epsilon$ ) such that

$$
\max _{M}\left|v_{\epsilon}\right| \leqq \max _{E_{\epsilon}}\left|v_{\epsilon}\right| \leqq K_{\epsilon}\left\|v_{\mathrm{e}}\right\|_{\mathrm{s}}
$$

Thus we have

$$
\max _{M}\left|v_{\epsilon}\right| \leqq K_{\epsilon} C_{8, \mathrm{e}}\|\bar{\partial} u\|_{s+1}
$$

8 The authors wish to thank Lars Hörmander for suggesting the method of proof in a letter to one of the authors. 
By appropriate choice of $u$ from the behavior of $C_{\boldsymbol{s}, \boldsymbol{\varepsilon}}$ and $K_{\mathrm{e}}$ it will follow that the right hand side tends to zero as $\epsilon$ tends to zero. This will show that we can uniformly approximate $f$ on $M$ by a function $h$ holomorphic in some $E_{e}$, but by using the fact that each $E_{e}$ is Runge with respect to some fixed $E_{\epsilon_{0}}, \epsilon_{0} \geqq \epsilon$, (see [2]), we obtain the desired result.

\section{REFERENCES}

1. E. Bishop, Uniform algebras, Proceedings of the Conference on Complex Analysis, Minneapolis, 1964, Springer-Verlag, Berlin, 1965.

2. H. J. Bremermann, Die Charakterisierung Rungescher Gebiete durch plurisubharmonische Funktionen, Math. Ann. 136 (1958), 173-186.

3. M. Freeman, "Some conditions for uniform approximation on a manifold," in Function Algebras, Scott, Foresman and Company, Chicago, Ill., 1965, pp. 42-65.

4. H. Grauert, On Levi's problem and the imbedding of real-analytic manifolds, Ann. of Math. 68 (1958), 460-472.

5. R. C. Gunning and H. Rossi, Analytic functions of several complex variables, Prentice-Hall, Englewood Cliffs, N. J., 1962.

6. J. J. Kohn, Harmonic integrals on strongly pseudo-convex manifolds. I, Ann. of Math. 78 (1963), 112-148.

7. — Harmonic integrals on strongly pseudo-convex manifolds. II, Ann. of Math. 79 (1963), 450-472.

8. - Boundaries of complex manifolds, Proceedings of the Conference on Complex Analysis, Minneapolis, 1964, Springer-Verlag, Berlin, 1965.

9. J. J. Kohn and L. Nirenberg, Non-coercive boundary value problems, Comm. Pure Appl. Math. 18 (1965), 443-492.

10. R. O. Wells, Jr., Holomorphic approximation on real-analytic submanifolds of a complex manifold, Proc. Amer. Math. Soc. 17 (1966), 1272-1275.

11. - Holomorphic hulls and holomorphic convexity of differentiable submanifolds, Trans. Amer. Math. Soc. (to appear).

12. J. Wermer, Uniform approximation and maximal ideal spaces, Bull. Amer. Math. Soc. 68 (1962), 298-305.

BRANDEIS UNIVERSITY AND

RICE UNIVERSITY 